\title{
Concordance and Discordance Between Brain Perfusion and Atrophy in Frontotemporal Dementia
}

\author{
Soichiro Shimizu • Yu Zhang • Joel Laxamana • \\ Bruce L. Miller • Joel H. Kramer • Michael W. Weiner • \\ Norbert Schuff \\ Published online: 23 February 2010 \\ (C) The Author(s) 2010. This article is published with open access at Springerlink.com
}

\begin{abstract}
The aim of this study was to determine if a dissociation between reduced cerebral perfusion and gray matter (GM) atrophy exists in frontotemporal dementia (FTD). The study included 28 patients with FTD and 29 cognitive normal $(\mathrm{CN})$ subjects. All subjects had MRI at $1.5 \mathrm{~T}$, including T1-weighted structural and arterial spin labeling (ASL) perfusion imaging. Non-parametric concordance/ discordance tests revealed that GM atrophy without hypoperfusion occurs in the premotor cortex in FTD whereas concordant GM atrophy and hypoperfusion changes are found in the right prefrontal cortex and bilateral medial frontal lobe. The results suggest that damage of brain function in FTD, assessed by ASL perfusion, can vary regionally despite widespread atrophy. Detection of discordance between brain perfusion and structure in FTD might aid diagnosis and staging of the disease.
\end{abstract}

Keywords Frontotemporal dementia · MRI · Cerebral blood flow - Gray matter atrophy · Statistical parametric mapping

S. Shimizu $(\bowtie) \cdot$ Y. Zhang $\cdot$ J. Laxamana $\cdot$ M. W. Weiner $\cdot$

N. Schuff $(\bowtie)$

Center for Imaging of Neurodegenerative Diseases,

Veterans Affairs Medical Center,

Department of Radiology and Biomedical Imaging,

University of California, San Francisco,

4150 Clement Street, 114M,

San Francisco, CA 94121, USA

e-mail: soichiro_s@hotmail.com

e-mail: Norbert.Schuff@va.gov

S. Shimizu $\cdot$ Y. Zhang $\cdot$ M. W. Weiner $\cdot$ N. Schuff Department of Radiology and Biomedical Imaging, University of California, San Francisco,

San Francisco, USA

B. L. Miller · J. H. Kramer

Department of Neurology, University of California, San Francisco,

San Francisco, USA

\section{Introduction}

Frontotemporal dementia (FTD) is a devastating progressive disorder characterized by impaired social and behavioral functioning. It is the third most common neurodegenerative disease after Alzheimer's disease (AD) and Lewy body disease (Neary et al. 1998). In general, FTD affects individuals at an earlier age than $\mathrm{AD}$ and also can progress more rapidly (McKhann et al. 2001). Many neuroimaging studies have been performed to identify affected brain regions in FTD and to help improve diagnostic accuracy. For example, positron emission tomography (PET) and single photon emission computed tomography (SPECT) studies have shown reduced metabolism and perfusion deficits in FTD, involving primarily the frontal lobe (Diehl et al. 2004; Grimmer et al. 2004; Ishii et al. 1998; Jeong et al. 2005; McMurtray et al. 2006; Pickut et al. 1997; Salmon et al. 2003). In addition, structural magnetic resonance imaging (MRI) studies, using regionally unbiased computational methods such as voxel-based morphometry (VBM), revealed widespread cortical atrophy also involving the frontal lobe and further including temporal lobe regions (Gee et al. 2003; Grossman et al. 2004; Rosen et al. 2002; Whitwell and Jack 2005; Whitwell et al. 2007, 2005). So far, however, the imaging studies in FTD have assessed functional and structural alterations separately, lacking the ability to explore relationships between these changes. In particular, functional alterations discordant to structural changes or vice versa might provide valuable information for accurate diagnosis and staging of the disease, in addition to concordant functional and structural alterations.

In general, brain function can be indexed by perfusion imaging. Arterial spin labeling (ASL) MRI measures brain perfusion entirely non-invasively (Roberts et al. 1994). This is accomplished by using the physical principles of MRI to magnetically label blood water as an endogenous tracer 
for blood flow. Furthermore, ASL-MRI can readily be performed in the same scan session as structural MRI. Previous ASL-MRI studies in FTD demonstrated frontal hypoperfusion, similar to findings using PET and SPECT (Du et al. 2006). Moreover, hypoperfusion in FTD remained significant even after accounting for brain atrophy and partial gray and white matter volume variations, implying that hypoperfusion can be independent of brain volume loss. However, the relationship between hypoperfusion and brain atrophy in FTD has not been investigated thoroughly.

Previously, our group developed a statistical method (Hayasaka et al. 2006) to identify cross-modality relationships (e.g. between perfusion and atrophy) without need to explicitly model the relations. This method has been used in MRI studies of $\mathrm{AD}$, revealing patterns of concordant as well as discordant regional alterations between cortical hypoperfusion and atrophy. Especially, discordant alterations of hypoperfusion without significant brain atrophy were found predominantly in frontal lobe regions in $\mathrm{AD}$ whereas atrophy without significant hypoperfusion involved primarily parietal brain regions. The findings imply that the pathological alterations in the $\mathrm{AD}$ brain that give rise to imaging abnormalities can be heterogeneous. Based on these observations in $\mathrm{AD}$, the overall goal of this study was to determine if similar concordant and discordant alterations between cortical hypoperfusion and atrophy can be detected in FTD.

\section{Method}

This cross-sectional study included 28 patients diagnosed with FTD and 29 cognitively normal $(\mathrm{CN})$ subjects. Of those, $21 \mathrm{FTD}$ and $25 \mathrm{CN}$ subjects were included in a previous publication ( $\mathrm{Du}$ et al. 2006). The FTD patients were referred from the Memory and Aging Clinic of the University of California, San Francisco (UCSF) while the control subjects were recruited from the community by posting flyers. All patients were diagnosed according to established consensus criteria (Neary et al. 1998) that were based upon information obtained from an extensive clinical history and physical examination. MRI was not exploited for the diagnosis of FTD but was used to rule out other major neuropathologies such as tumors, stroke, severe white matter disease, or inflammation. Patients and controls were included in the study if they were between 35 and 80 years old and had no history of brain trauma, brain tumor, stroke, epilepsy, alcoholism, psychiatric illness, or a systemic disease that affects brain function (e.g. hypothyroidism). None of the patients had a family history of FTD. Furthermore, only FTD patients without motor neuron disease-related symptoms, were included.
All subjects received a standard battery of neuropsychological tests, as described in detail before (Rosen et al. 2004). In brief, the tests included assessment of global cognitive impairment by the Mini-Mental State Examination (MMSE) (Folstein et al. 1975), assessment of global functional impairment by the Clinical Dementia Rating (CDR) scale (Morris 1993) and assessment of executive functions by a modified version of the Trail-Making Test (TMT) (Rosen et al. 2004). All subjects or their legal guardians gave written informed consent before participating in the study, which was approved by the Committees of Human Research of the University of California and the VA Medical Center in San Francisco.

\section{MRI acquisition and processing}

All subjects were scanned using a Siemens $1.5 \mathrm{~T}$ Vision MRI system (Siemens Inc, Erlangen, Germany) equipped with a quadrature channel head coil. T1-weighted structural images were acquired using a magnetization prepared rapid acquisition gradient-echo (MPRAGE) sequence with repetition (TR), echo (TE) and inversion (TI) timings of 9/ $4 / 300 \mathrm{~ms}$, respectively, $15^{\circ}$ flip angles, $1.0 \times 1.0 \mathrm{~mm}^{2}$ in plane resolution based on a $256 \times 256$ acquisition matrix and 154 coronal slices, each $1.5 \mathrm{~mm}$ thick. In addition, perfusion weighted images (PWI) were acquired using the concept of pulsed arterial spin labeling by double inversions with proximal labeling of both tag and control images (DIPLOMA) as described in reference (Jahng et al. 2003). The parameters of PWI were TR/TE $=2,500 / 15 \mathrm{~ms}$ with a post-labeling delay of $1,500 \mathrm{~ms}$ before the PWI signal was mapped using single shot echo planar imaging (EPI). The inplane resolution of EPI was $2.3 \times 2.3 \mathrm{~mm}^{2}$ based on a $128 \times 128$ acquisition matrix. For each perfusion scan, 5 slices each $8 \mathrm{~mm}$ thick and $2 \mathrm{~mm}$ gap covering the brain regions above the AC-PC line were acquired. More details about the PWI acquisition are described in Johnson et al. (2005).

All images were processed as described in detail previously (Johnson et al. 2005). In short, each PWI was co-registered to a reference EPI (without labeling), which in turn was co-registered to the corresponding T1-weighted image using affine transformations with 9 degrees of freedom and the normalized mutual information coregistration algorithm available in the Statistical Parametric Mapping (SPM2) program (Welcome Department of Imaging Neuroscience; London, UK). Furthermore, the T1-weighted images were segmented into probabilistic maps of gray matter (GM), white matter (WM) and cerebrospinal fluid (CSF) using Expectation-Maximization Segmentation (EMS), also available as add-on in SPM2 (Van Leemput et al. 1999, 2001). Although we did not 
express $\mathrm{CBF}$ in absolute units, the differences between the two groups are still valid, especially since we accounted for global variations in CBF across the subjects.

Then the probabilistic tissue maps were blurred to the resolution of PWI to estimate the GM, WM and CSF content of each voxel in the corresponding PWI image. Partial volume effect (PVE) correction for CSF was calculated for each voxel on the perfusion image on the basis of the method by Müller-Gärtner et al. (1992), which assumes that all contributions to perfusion are from brain tissue and that CSF contributes nothing. Furthermore, to account for variable amounts of GM and WM in the voxels, it was assumed that perfusion of GM is 2.5 times the perfusion of WM (Roberts et al. 1994), leading to corrected PWI intensities according to: $\mathrm{SI}_{\text {corr }}=\mathrm{SI}_{\text {uncorr }} /(\mathrm{GM}+$ $0.4 * \mathrm{WM})$, where $\mathrm{SI}_{\text {corr }}$ and $\mathrm{SI}_{\text {uncorr }}$ are the corrected and uncorrected perfusion signal intensities, respectively, and GM and WM are the corresponding probabilities of gray or white matter volumes, respectively, with $\mathrm{GM}+\mathrm{WM}+\mathrm{CSF}=1$. To constrain the analysis to GM perfusion (since the PWI signal of WM is usually very small and CSF contributes only noise), a threshold was applied that included only voxels with at least $75 \%$ gray matter and less than $25 \%$ other brain tissue, such as white matter and cerebrospinal fluid. Finally, to reduce high signal artifacts in PWI (i.e. signal from blood vessels), PWI signals above two standard deviations of the mean signal were capped to a maximum of two standard deviations above the $\mathrm{SI}_{\text {corr }}$ For voxelwise tests the T1-weighted images were spatially normalized to a study-specific brain template. The study-specific template was created by averaging the spatially normalized T1-weighted images of all subjects in the study using an initial affine transformation with 12 degrees of freedom, followed by non-linear transformations with $10 \times 9 \times 10$ discrete cosine basis functions. To test for regional brain atrophy, the T1-weighted images also were segmented, smoothed (6 $\mathrm{mm}$ isotropic Gaussian), and modulated according to the optimized VBM protocol as outlined by Good et al. (2001).

To test for regional hypoperfusion, the PWI images were normalized to the T1-weighted template space by applying the same transformation parameters than for the T1-weighted images. In addition, the PWI images were re-sliced to $2 \times 2 \times$ $2 \mathrm{~mm}$ voxel size and smoothed with a 12-mm Gaussian kernel to account for imperfection in co-registration.

\section{Statistical analysis}

Initially, voxelwise tests of differences in GM atrophy or GM perfusion between FTD and $\mathrm{CN}$ subjects were performed separately using two sample t-tests. For GM atrophy the t-tests were further augmented by an analysis of co-variance (ANCOVA) with adjustments for variations in age and total intra-cranial volumes. Similarly, for hypoperfusion the t-tests were augmented by ANCOVA with adjustments for variations in age and global perfusion to account for global variations in blood flow between subjects. The level of significance for these tests was set at $p=0.05$, including adjustments for multiple comparisons based on the concept of false discovery rates (FDR) (Genovese et al. 2002).

Following the t-tests, a concordant/discordant analysis was performed. For the concordant/discordant analysis of GM atrophy and hypoperfusion changes, we used a nonparametric multimodal correlation test as described in our previous report (Hayasaka et al. 2006). In brief, this test performs a statistic on the T-values of both GM atrophy, denoted here $\mathrm{S}_{\mathrm{GM}}$ and T-values of hypoperfusion, denoted $\mathrm{S}_{\text {Perf }}$. The analysis includes first, the design of combining functions of concordance or discordance between GM atrophy and hypoperfusion, followed by permutation tests to obtain the distribution of each combining function and to perform a statistic.

We defined the following combining functions:

(1) Concordance function: $\mathrm{W}_{\mathrm{CON}}=\mathrm{S}_{\mathrm{GM}} \times \mathrm{S}_{\text {perf }}$, identifies regions of concordance between GM atrophy and hypoperfusion. For statistical inference of the concordance function, we set the critical threshold of Tvalues for concordance to a value equivalent to the joint distribution of atrophy and perfusion at $p<0.05$ statistical significance with a family-wise error (FWE) adjustment for multiple comparisons, as illustrated in Fig. 1.

(2) Discordance functions: $\mathrm{W}_{\mathrm{DIS}}=\mathrm{S}_{\text {perf }}-\left(\lambda \times \mathrm{S}_{\mathrm{GM}}\right)^{2 \eta}$ identifies regions with changes in GM perfusion without substantial GM atrophy. Here, $\lambda$ defines the width and $\eta$ the base of the parabolic function. Conversely, $\mathrm{W}_{\mathrm{DIS}}=\mathrm{S}_{\mathrm{GM}}-\left(\lambda \times \mathrm{S}_{\mathrm{perf}}\right)^{2 \eta}$ identifies regions with changes in GM atrophy without substantial hypoperfusion. We chose parabolic functions to impose smooth boundaries for critical regions of discordance. Furthermore, we chose $\lambda=0.5$ so that critical regions of discordance roughly span a range from $-2 \leq \mathrm{T} \geq 2$ and a typical t-test fails to reject the null hypothesis at $p=$ 0.05 in such regions. Also we chose $\eta=2$ to be wider than a typical parabola (i.e. $\eta=1$ ) to increase the sensitivity in the critical regions. More details about these functions and settings are described in Hayasaka et al. (2006) and Zhang et al. (2008).

Figure 1 shows a scatter plot of T-values of GM perfusion versus GM atrophy from a t-test comparing FTD to $\mathrm{CN}$ subjects. Also illustrated are the various combining functions at a statistical threshold of $p=0.05$ (FWE). 
Fig. 1 Scatter plot of t-values of gray matter hypoperfusion versus gray matter atrophy from a t-test comparing FTD patients with control subjects. The cluster defining thresholds of the combining functions at $p=0.05$ (adjusted for family-wise errors) are indicated and described in the figure legend

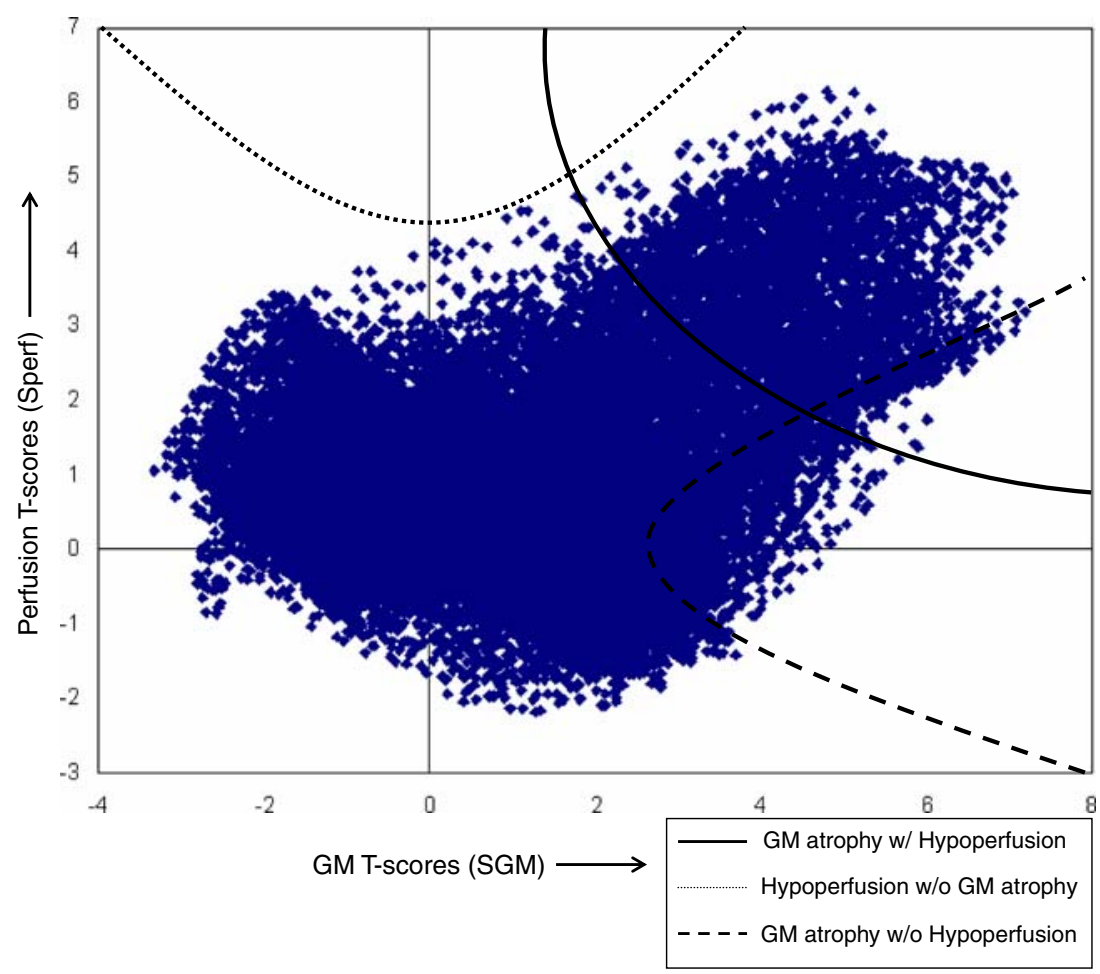

To determine the statistical distribution of voxels defined by each combining function, we performed permutation tests on the group of subjects and used the cluster mass of voxels as a test statistic. These tests were conducted using the Statistical non-Parametric Mapping (SnPM) package (http://www.sph.umich.edu/ni-stat/SnPM).

The level of statistical significance in imaging tests was set to $p<0.05$. We used the concept of false discovery rate (FDR) (Genovese et al. 2002) to account for multiple comparisons in separate imaging tests of brain atrophy and hypoperfusion. To account for multiple comparisons in concordance/discordance tests of brain atrophy and hypoperfusion, we used adjustments based on FWE, which are more stringent than those based on FDR.

P-values less than the threshold were then linked to their corresponding anatomical localizations and superimposed on a brain map.

\section{Result}

Table 1 lists the demographics and clinical data of the study population. This shows a trend for FTD patients being moderately younger than control subjects $(p=0.06)$. The groups did not differ significantly by gender $\left(\chi^{2}=0.3, p=\right.$ 0.5 ). As expected, FTD patients scored significantly worse on MMSE $(p<0.001)$, CDR $(p<0.001)$ and modified trials tests $(p<0.001)$ than $\mathrm{CN}$ subjects.

Figure 2 shows the results from separate SPM group analyses of GM hypoperfusion (A) and GM atrophy (B) in
FTD relative to $\mathrm{CN}$ subjects. Only regions that both T1weighted and ASL perfusion MRI covered are shown. This indicates FTD patients had significant hypoperfusion bilaterally in the medial frontal cortex and right prefrontal cortex $(p<0.05$, FDR corrected). At the same statistical threshold level than perfusion, GM atrophy was by far more widespread in FTD, involving bilaterally large aspects of the medial frontal lobe and extending into the temporal lobes $(p<0.05, \mathrm{FDR})$.

Figure 3 shows results from a concordance analysis of reduced GM perfusion and GM atrophy in FTD relative to controls. Concordance was found in the right prefrontal cortex and bilateral medial frontal lobe $(p<0.05$, FWE corrected).

Figure 4 shows results from a discordance analysis of GM atrophy without significant hypoperfusion between FTD patients and control subjects, involving bilaterally the premotor cortex $(p<0.05)$. A discordance analysis of hypoperfusion without significant GM atrophy yielded no significant region.

\section{Discussion}

The main finding of this study is that FTD was associated with discordant cortical atrophy in absence of significant reduction of perfusion in premotor areas whereas concordant alterations of cortical atrophy and hypoperfusion occurred predominantly in the right prefrontal cortex and bilaterally in the medial frontal lobe. The new finding in 
Table 1 Demographic and clinical data of the patients with FTD and control $(\mathrm{CN})$ subjects

\begin{tabular}{llll}
\hline Demographics & $\operatorname{FTD}(n=28)$ & $\mathrm{CN}(n=29)$ & $\mathrm{P}$ values \\
\hline Mean age (years) & $58.9 \pm 8.4$ & $63.8 \pm 11.1$ & 0.06 \\
Men/Women & $17 / 11$ & $15 / 14$ & 0.5 \\
MMSE (scale 0-30) & $23.0 \pm 7.2$ & $29.7 \pm 0.8$ & $<0.001$ \\
CDR box scores (scale 0-18) & $7.5 \pm 3.8$ & $0 \pm 0$ & $<0.001$ \\
Modified trials (scale 0-infinity) $^{\mathrm{c}}$ & $14.2 \pm 14.0$ & $34.2 \pm 19.5$ & $<0.001$ \\
\hline
\end{tabular}

Values are mean \pm standard deviation

${ }^{a}$ MMSE: Mini Mental State Examination, decreasing scores indicating increasing cognitive impairment

${ }^{\mathrm{b}}$ CDR: Clinical Dementia Rating score with increasing scores of sum of boxes indicating increasing cognitive impairment

${ }^{\mathrm{c}}$ Modified trails score indicating number of lines drawn per minute and hence a lower score indicating increased impairment

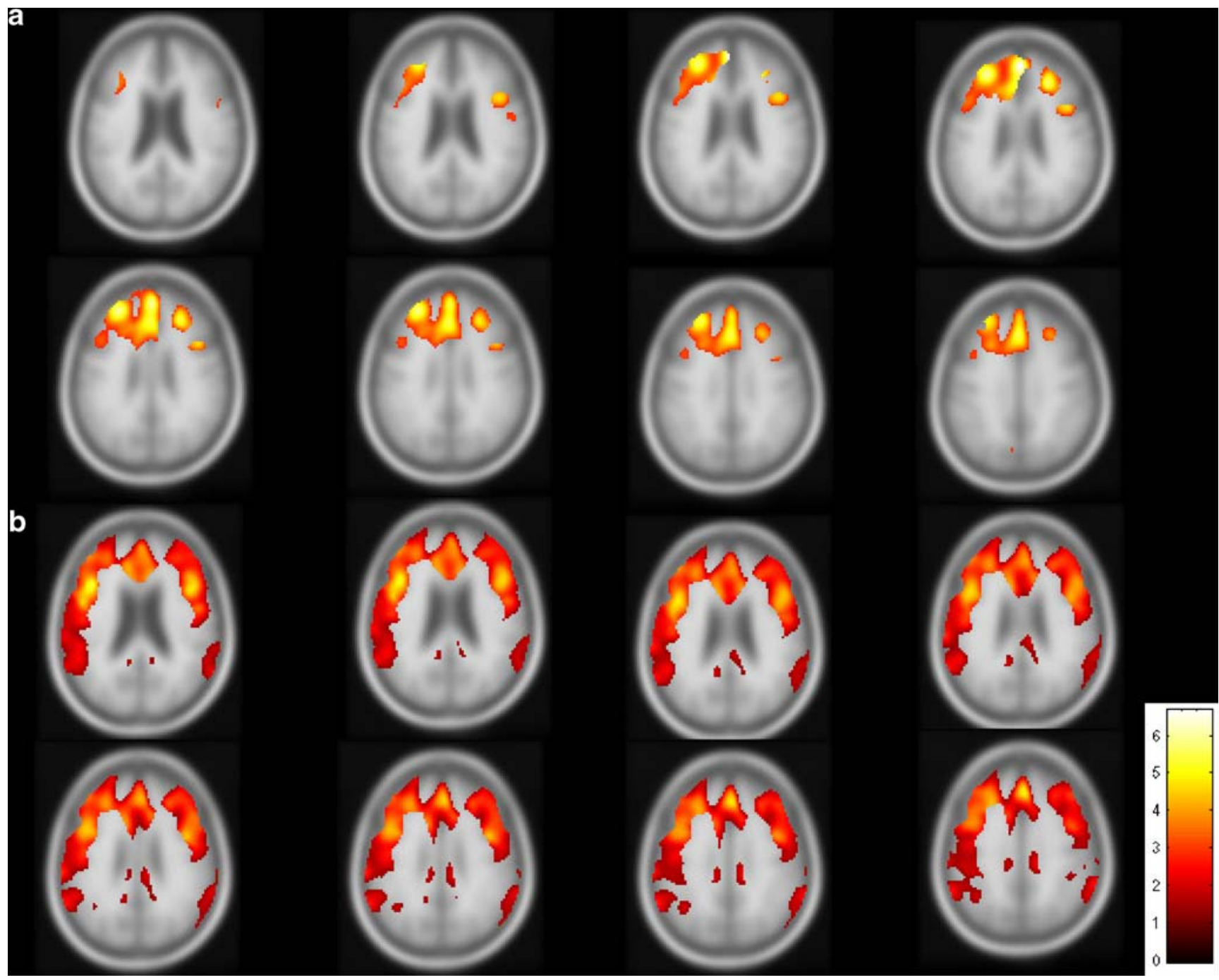

Fig. 2 Results, showing significant T-values from separate analyses of GM atrophy (a) and hypoperfusion (b) in patients with frontotemporal dementia (FTD) compared to control subjects $(p<0.05$, FDR corrected). The color scale of T-values is indicated in the lower right corner. Only regions that were covered by both T1-weighted and ASL perfusion MRI are shown 


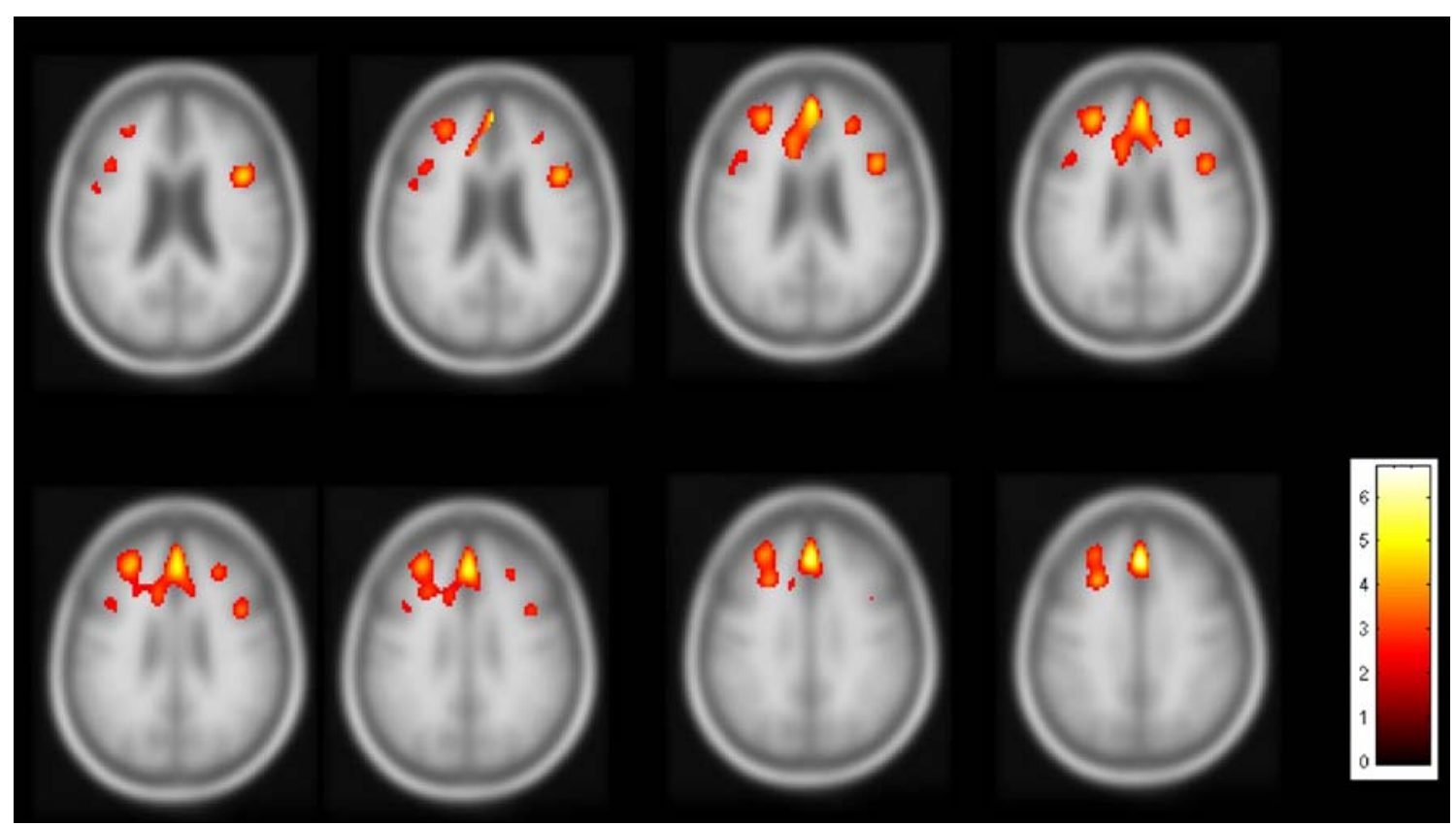

Fig. 3 Results from a concordance analysis of both gray matter atrophy and hypoperfusion in FTD relative to control subjects. Clusters of significant concordance are indicated $(p<0.05$, FWE corrected)

this study is the detection of concordance/discordance regions between hypoperfusion and GM atrophy in patients with FTD, compared to a previous report involving largely the same patients that showed only hypoperfusion. In addition, tests of cortical atrophy and hypoperfusion in FTD when conducted separately indicated widespread atrophy involving large aspects of the lateral and medial frontal lobe bilaterally and hypoperfusion in the medial frontal and right prefrontal cortex, consistent with previous imaging reports (Broe et al. 2003; Diehl et al. 2004; Du et al. 2007; Gee et al. 2003; Grimmer et al. 2004; Grossman et al. 2004; Ishii et al. 1998; Jeong et al. 2005; McMurtray

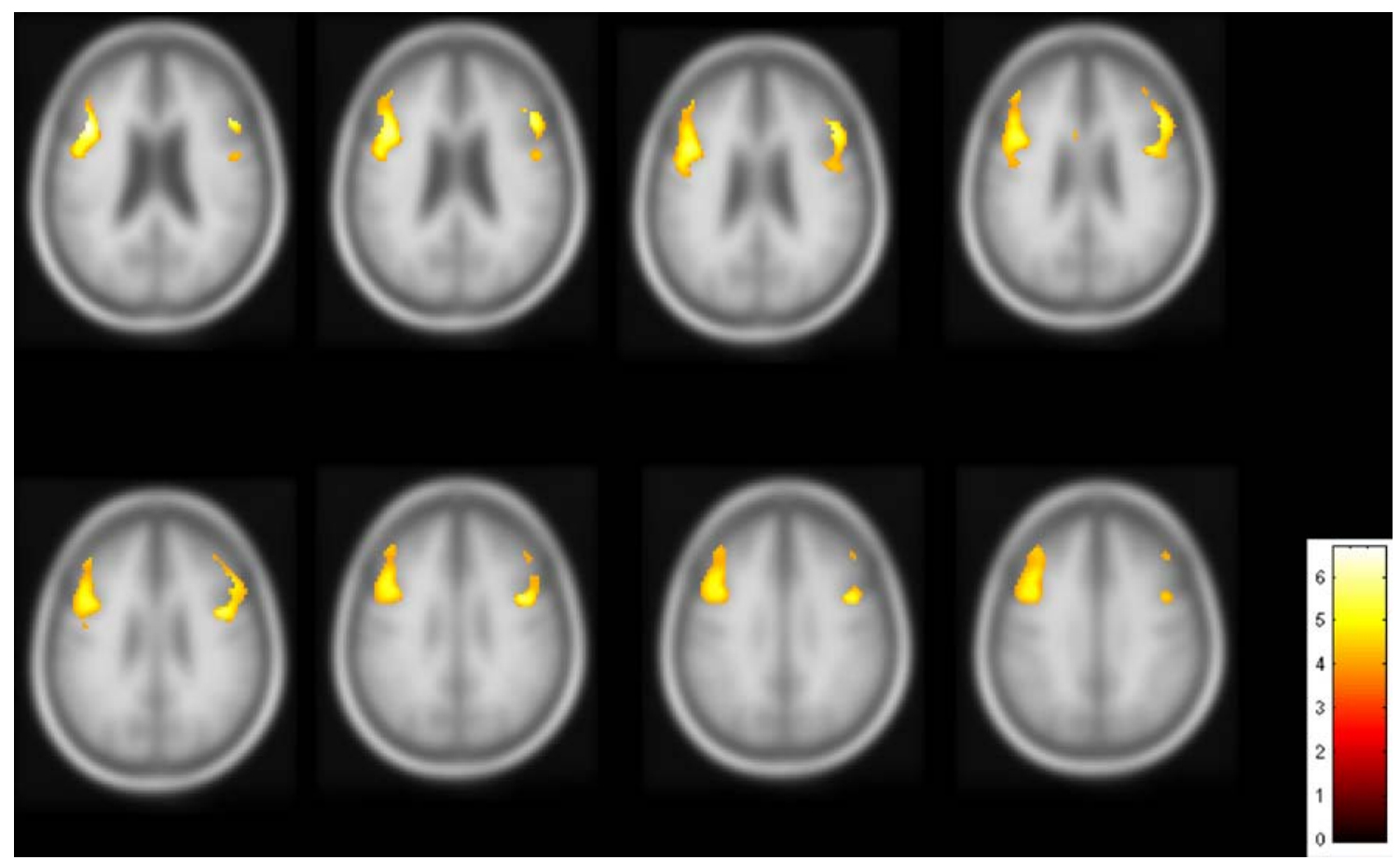

Fig. 4 Results from a discordance analysis of gray matter atrophy without significant hypoperfusion in FTD relative to control subjects. Clusters of significant dissociation are shown $(p<0.05$ FWE corrected) 
et al. 2006; Pickut et al. 1997; Rosen et al. 2002; Salmon et al. 2003; Whitwell and Jack 2005; Whitwell et al. 2005, 2007).

We interpret the finding of discordant brain atrophy in absence of hypoperfusion in the premotor cortex as indication of brain tissue that is still functioning and connected in FTD, though other explanations are possible: First, loss of brain tissue other than neurons might contribute disproportionately to atrophy while surviving neurons still function normally as reflected by normal levels of perfusion. Indeed, some histopathological studies suggest that earliest cellular changes in FTD occur in astrocytes without frank neuron loss whereas neuronal damage is found in later stages of the disease (Broe et al. 2004; Kersaitis et al. 2004). This view is also supported by clinical observations that many patients with FTD do not have motor symptoms, despite apparent atrophy of the premotor cortex. Discordant MRI perfusion in presence of brain atrophy might provide an index for the condition of motor neurons in FTD which atrophy alone cannot provide. The recruited patients with FTD were all mild to moderate stage and accounting for disease severity, e.g. using MMSE, did not significantly alter the results. However, given the small number of subjects and limited power, we cannot exclude that the results might change for patients at a more advanced stage of FTLD. Further studies permitting concurrent assessment of structural and function alterations in FTD are necessary to test this conjecture.

Second, it is also possible that we lacked power with ASL-MRI to measure small alterations in hypoperfusion in presence of GM atrophy. A conservative power analysis of our data based on previous reliability tests (Jahng et al. 2005) suggests that the minimum difference in perfusion we can expect to detect with 28 patients and controls and $80 \%$ power (alpha $=0.05$ ) is about $20 \%$. In comparison, the minimum difference in atrophy that we can detect at the same level of power and significance is $1.5 \%$. This may also explain why we did not observe significant discordance between hypoperfusion without GM atrophy in FTD. In another study involving $\mathrm{AD}$, however, we found regions with significant hypoperfusion in absence of atrophy, indicating that it is principally possible to measure discordant alterations between atrophy and perfusion with our method. Another issue that suggests technology rather than biological effects explains discrepancies between structural and perfusion findings are the typical observation of higher SNR of the perfusion signal from the frontal cortex, where regions are more adjacent to one another, compared to SNR of the perfusion signal from parietal brain regions. Hence, differences will be more datable in frontal than parietal brain regions. We also found concordant reductions of GM volume and perfusion in the right prefrontal cortex and bilateral medial frontal lobe, regions that have been consistently implicated in the pathology of FTD. Since the perfusion data were corrected for CSF and $\mathrm{GM} / \mathrm{WM}$ tissue variations, the concurrent reductions in perfusion and GM volume cannot be explained simply as an artifact of partial volume effects. One possible explanation for concordant atrophy and hypoperfusion is that the remaining brain tissue is already damaged as indicated by reduced perfusion. It is interesting that the regions showing concurrent atrophy and hypoperfusion involved the right prefrontal cortex and bilaterally the medial frontal lobe. These regions are considered particularly vulnerable to FTD, as indicated in previous imaging studies (Broe et al. 2003). In addition, we found that concordance regions in the predominant right frontal lobe. It is consistent with several studies (Ishii et al. 1998; McMurtray et al. 2006; $\mathrm{Du}$ et al. 2006) that showed hypoperfusion and hypometabolism in the predominant right frontal lobe. The clinical significance of the metabolic and perfusion asymmetry is unknown. One possible explanation may be caused by sampling bias. Left-dominant patients can show language disturbances even in early stages. Thus, it is more likely that these patients are brought to neurology clinics. On the other hand, right-dominant patients may present predominantly with behavioral or psychiatric abnormalities, and so are first seen as psychiatric abnormalities, and potentially are misdiagnosed as having psychiatric illnesses. It should be noted that the equivalence between structural MRI and functional imaging, i.e. FDG-PET, in FTD has been seen in multiple studies of the two techniques independently but also in studies comparing the two modalities (Kanda et al. 2008; Kipps et al. 2009). Therefore, our findings of concordance, which agree with these previous studies, are not surprising. Nonetheless, the results support the equivalence between ASL measured hypoperfusion and PET measured hypometabolism in FTD.

The image analysis approach used in this report was previously employed to explore the concordance/discordance between structural and perfusion changes in patients with $\mathrm{AD}$ and mild cognitive impairment (Hayasaka et al. 2006). Our results demonstrate that the same concept applied to FTD provides new information, not available from testing each modality separately. The method could potentially be used to explore the relationship between various other imaging modalities, including PET and SPECT, in FTD and other neurodegenerative diseases.

Several limitations in our study ought to be mentioned. First, the patients were diagnosed clinically, without autopsy confirmation of FTD. Thus, it is possible that some of patients with FTD had other causes of dementia. Studies involving brain autopsies are needed to confirm that our findings are related to FTD pathology. The small number of patients limits power to detect significant 
differences in perfusion and generalization of the findings. Further studies including a larger number of patients are warranted to confirm the findings. A technical limitation is that this implementation of ASL-MRI in conjunction with the short lifetime of spin labels at $1.5 \mathrm{~T}$ did not permit covering more brain regions, especially the temporal lobes, which are also susceptible to FTD. Therefore, we may have missed other prominent regions with atrophy and hypoperfusion in FTD. Lack of perfusion data from the ventral prefrontal cortex and most of the temporal lobes limit, which both can be highly impacted in FTLD, limit the conclusions in this study. New volumetric ASL-MRI methods (Fernández-Seara et al. 2005; Günther et al. 2005) can provide more brain coverage, including the temporal lobe, especially when performed at a higher magnetic field strength than $1.5 \mathrm{~T}$. Finally, another technical limitation is that the choice of combining functions is somewhat arbitrary and subjective and different choices for combining functions might alter the outcome. However, the sensitivity and robustness of the combining functions can be evaluated by simulations and permutation tests as we previously showed (Hayasaka and Nichols 2004). In addition, the functions we chose here are identical to those we used in previous studies of Alzheimer's disease (Hayasaka et al. 2006) and brain aging (Zhang et al. 2008). Another limitation is that our concordance/discordance analysis is constraint to an analysis at the group level while examinations at the single subject level might be even more informative. In theory, a potential solution to this is performing the concordance/discordance analysis using each subject's regional z-scores (deviation from the normal mean) rather than $t$-scores from the group comparison, supplemented by an appropriate statistic that takes both between group and across subject variations into account.

In summary, we found concordant hypoperfusion and brain atrophy in the right prefrontal cortex and bilateral medial frontal lobe in FTD as well as discordant atrophy without significant hypoperfusion in premotor regions. These results suggest that damage of brain function in FTD may vary regionally despite widespread atrophy. Detection of discordance between brain perfusion and structure in FTD might aid diagnosis and staging of the disease.

Acknowledgement This study was supported in part by NIH grant (P01AG019724) and a grant from the National Center for Research Resources (P41 RR023953). This material is also the result of work supported with resources and the use of facilities at the Veterans Affairs Medical Center in San Francisco.

Open Access This article is distributed under the terms of the Creative Commons Attribution Noncommercial License which permits any noncommercial use, distribution, and reproduction in any medium, provided the original author(s) and source are credited.

\section{References}

Broe, M., Hodges, J. R., Schofield, E., Shepherd, C. E., Kril, J. J., \& Halliday, G. M. (2003). Staging disease severity in pathologically confirmed cases of frontotemporal dementia. Neurology, 60(6), $1005-1011$.

Broe, M., Kril, J., \& Halliday, G. M. (2004). Astrocytic degeneration relates to the severity of disease in frontotemporal dementia. Brain, 127, 2214-2220. doi:10.1093/brain/awh250.

Diehl, J., Grimmer, T., Drzezga, A., Riemenschneider, M., Förstl, H., \& Kurz, A. (2004). Cerebral metabolic patterns at early stages of frontotemporal dementia and semantic dementia. A PET study. Neurobiology of Aging, 25(8), 1051-1056. doi:10.1016/j.neuro imaging.2003.10.007.

Du, A. T., Jahng, G. H., Hayasaka, S., Kramer, J. H., Rosen, H. J., Gorno-Tempini, M. L., et al. (2006). Hypoperfusion in frontotemporal dementia and Alzheimer disease by arterial spin labeling MRI. Neurology, 67(7), 1215-1220.

Du, A. T., Schuff, N., Kramer, J. H., Rosen, H. J., Gorno-Tempini, M. L., Rankin, K., et al. (2007). Different regional patterns of cortical thinning in Alzheimer's disease and frontotemporal dementia. Brain, 130, 1159-1166. doi:10.1093/brain/awm016.

Fernández-Seara, M. A., Wang, Z., Wang, J., Rao, H. Y., Guenther, M., Feinberg, D. A., et al. (2005). Continuous arterial spin labeling perfusion measurements using single shot 3D GRASE at 3 T. Magnetic Resonance in Medicine, 54(5), 1241-1247.

Folstein, M. F., Folstein, S. E., \& McHugh, P. R. (1975). Mini-mental state. A practical method for grading the cognitive state of patients for the clinician. Journal of Psychiatric Research, 12, 189-198.

Gee, J., Ding, L., Xie, Z., Lin, M., DeVita, C., \& Grossman, M. (2003). Alzheimer's disease and frontotemporal dementia exhibit distinct atrophy-behavior correlates: a computer-assisted imaging study. Academic Radiology, 10(12), 1392-1401.

Genovese, C. R., Lazar, N. A., \& Nichols, T. (2002). Thresholding of statistical maps in functional neuroimaging using the false discovery rate. Neuroimage, 15(4), 870-878.

Good, C. D., Johnsrude, I. S., Ashburner, J., Henson, R. N., Friston, K. J., \& Frackowiak, R. S. (2001). A voxel-based morphometric study of ageing in 465 normal adult human brains. Neuroimage, 14, 21-36. doi:10.1006/nimg.2001.0786.

Grimmer, T., Diehl, J., Drzezga, A., Förstl, H., \& Kurz, A. (2004). Region-specific decline of cerebral glucose metabolism in patients with frontotemporal dementia: a prospective ${ }^{18} \mathrm{~F}-\mathrm{FDG}-$ PET study. Dementia and Geriatric Cognitive Disorders, 18(1), 32-36. doi:10.1159/000077732.

Grossman, M., McMillan, C., Moore, P., Ding, L., Glosser, G., Work, M., et al. (2004). What's in a name: voxel-based morphometric analyses of MRI and naming difficulty in Alzheimer's disease, frontotemporal dementia and corticobasal degeneration. Brain, 127(Pt 3), 628-649. doi:10.1093/brain/awh075.

Günther, M., Oshio, K., \& Feinberg, D. A. (2005). Single-shot 3D imaging techniques improve arterial spin labeling perfusion measurements. Magnetic Resonance in Medicine, 54(2), 491-498.

Hayasaka, S., \& Nichols, T. E. (2004). Combining voxel intensity and cluster extent with permutation test framework. Neuroimage, 23 (1), 54-63.

Hayasaka, S., Du, A. T., Duarte, A., Kornak, J., Jahng, G. H., Weiner, M. W., et al. (2006). A non-parametric approach for co-analysis of multi-modal brain imaging data: application to Alzheimer's disease. Neuroimage, 30(3), 768-779. doi:10.1016/j.neuroimage. 2005.10.052.

Ishii, K., Sakamoto, S., Sasaki, M., Kitagaki, H., Yamaji, S., Hashimoto, M., et al. (1998). Cerebral glucose metabolism in 
patients with frontotemporal dementia. Journal of Nuclear Medicine, 39(11), 1875-1878.

Jahng, G. H., Zhu, X. P., Matson, G. B., Weiner, M. W., \& Schuff, N. (2003). Improved perfusion-weighted MRI by a novel double inversion with proximal labeling of both tagged and control acquisitions. Magnetic Resonance in Medicine, 49(2), 307-314.

Jahng, G. H., Song, E., Zhu, X. P., Matson, G. B., Weiner, M. W., \& Schuff, N. (2005). Human brain: reliability and reproducibility of pulsed arterial spin-labeling perfusion MR imaging. Radiology, 234(3), 909-916.

Jeong, Y., Cho, S. S., Park, J. M., Kang, S. J., Lee, J. S., Kang, E., et al. (2005). ${ }^{18}$ F-FDG PET findings in frontotemporal dementia: an SPM analysis of 29 patients. Journal of Nuclear Medicine, 46(2), 233-239.

Johnson, N. A., Jahng, G. H., Weiner, M. W., Miller, B. L., Chui, H. C., Jagust, W. J., et al. (2005). Pattern of cerebral hypoperfusion in Alzheimer disease and mild cognitive impairment measured with arterial spin-labeling MR imaging: initial experience. Radiology, 234(3), 851-859.

Kanda, T., Ishii, K., Uemura, T., Miyamoto, N., Yoshikawa, T., Kono, A. K., et al. (2008). Comparison of grey matter and metabolic reductions in frontotemporal dementia using FDG-PET and voxel-based morphometric MR studies. European Journal of Nuclear Medicine and Molecular Imaging, 35(12), 2227-2234.

Kersaitis, C., Halliday, G. M., \& Kril, J. J. (2004). Regional and cellular pathology in frontotemporal dementia: relationship to stage of disease in cases with and without Pick bodies. Acta Neuropathologica, 108(6), 515-523. doi:10.1007/s00401-004-0917-0.

Kipps, C. M., Hodges, J. R., Fryer, T. D., \& Nestor, P. J. (2009). Combined magnetic resonance imaging and positron emission tomography brain imaging in behavioural variant frontotemporal degeneration: refining the clinical phenotype. Brain, 132(Pt 9), 2566-2578.

McKhann, G. M., Albert, M. S., Grossman, M., Miller, B., Dickson, D., Trojanowski, J. Q., et al. (2001). Clinical and pathological diagnosis of frontotemporal dementia: report of the Work Group on Frontotemporal Dementia and Pick's Disease. Archives of Neurology, 58(11), 1803-1809.

McMurtray, A. M., Chen, A. K., Shapira, J. S., Chow, T. W., Mishkin, F., Miller, B. L., et al. (2006). Variations in regional SPECT hypoperfusion and clinical features in frontotemporal dementia. Neurology, 66(4), 517-522.

Morris, J. C. (1993). The Clinical Dementia Rating (CDR): current version and scoring rules. Neurology, 43, 2412-2414.

Müller-Gärtner, H. W., Links, J. M., Prince, J. L., Bryan, R. N., McVeigh, E., Leal, J. P., et al. (1992). Measurement of radiotracer concentration in brain gray matter using positron emission tomography: MRI-based correction for partial volume effects. Journal of Cerebral Blood Flow and Metabolism, 12(4), 571-583.
Neary, D., Snowden, J. S., Gustafson, L., et al. (1998). Frontotemporal lobar degeneration: a consensus on clinical diagnostic criteria. Neurology, 51, 1546-1554.

Pickut, B. A., Saerens, J., Mariën, P., Borggreve, F., Goeman, J., Vandevivere, J., et al. (1997). Discriminative use of SPECT in frontal lobe-type dementia versus (senile) dementia of the Alzheimer's type. Journal of Nuclear Medicine, 38(6), 929-934.

Roberts, D. A., Detre, J. A., Bolinger, L., Insko, E. K., \& Leigh, J. S., Jr. (1994). Quantitative magnetic resonance imaging of human brain perfusion at $1.5 \mathrm{~T}$ using steady-state inversion of arterial water. Proceedings of the National Academy of Sciences of the United States of America, 91, 33-37.

Rosen, H. J., Gorno-Tempini, M. L., Goldman, W. P., Perry, R. J., Schuff, N., Weiner, M., et al. (2002). Patterns of brain atrophy in frontotemporal dementia and semantic dementia. Neurology, 58 (2), 198-208.

Rosen, H. J., Naravaez, J. M., Hallam, B., et al. (2004). Neuropsychological and functional measures of severity in Alzheimer disease, frontotemporal dementia, and semantic dementia. Alzheimer Disease and Associated Disorders, 18, 202-207.

Salmon, E., Garraux, G., Delbeuck, X., Collette, F., Kalbe, E., Zuendorf, G., et al. (2003). Predominant ventromedial frontopolar metabolic impairment in frontotemporal dementia. Neuroimage, 20(1), 435-440. doi:10.1016/S1053-8119(03)00346-X.

Van Leemput, K., Maes, F., Vandermeulen, D., \& Suetens, P. (1999). Automated model-based tissue classification of MR images of the brain. IEEE Transactions on Medical Imaging, 18(10), 897908.

Van Leemput, K., Maes, F., Vandermeulen, D., Colchester, A., \& Suetens, P. (2001). Automated segmentation of multiple sclerosis lesions by model outlier detection. IEEE Transactions on Medical Imaging, 20(8), 677-688.

Whitwell, J. L., \& Jack, C. R., Jr. (2005). Comparisons between Alzheimer disease, frontotemporal lobar degeneration, and normal aging with brain mapping. Topics in Magnetic Resonance Imaging, 16(6), 409-425.

Whitwell, J. L., Josephs, K. A., Rossor, M. N., Stevens, J. M., Revesz, T., Holton, J. L., et al. (2005). Magnetic resonance imaging signatures of tissue pathology in frontotemporal dementia. Archives of Neurology, 62(9), 1402-1408.

Whitwell, J. L., Jack, C. R., Jr., Baker, M., Rademakers, R., Adamson, J., Boeve, B. F., et al. (2007). Voxel-based morphometry in frontotemporal lobar degeneration with ubiquitin-positive inclusions with and without progranulin mutations. Archives of Neurology, 64 (3), 371-376.

Zhang, Y., Du, A. T., Hayasaka, S., Jahng, G. H., Hlavin, J., Zhan, W., et al. (2008). Patterns of age-related water diffusion changes in human brain by concordance and discordance analysis. Neurobiology of Aging, doi:10.1016/j.neurobiolaging.2008.10.009. 